\title{
A SITUAÇÃO DA ECONOMIA DO ESPÍRITO SANTO NO INÍ́CIO DO SÉCULO XXI: UM ESTADO DESENVOLVIDO E PERIFERICO?
}

The state of the economy of Espírito Santo at the beginning of the 21st century: a developed and peripheral state?

El estado de la economía del Espírito Santo al principio del siglo XXI: ¿un estado desarrollado y periférico?

Sávio Bertochi Caçador

Mestre em Economia pela UFES e Economista do Bandes e-mail: sbcacador@hotmail.com

Robson Antonio Grassi Doutor em Economia pelo IE/UFRJ. Professor do Departamento e do Mestrado em Economia da UFES

e-mail: ragrassi@uol.com.br

\section{Resumo}

O objetivo do artigo é mostrar o caráter contraditório da condição da economia capixaba nos primeiros anos do presente século: uma economia com indicadores que revelam ao mesmo tempo certo grau de desenvolvimento socioeconômico e inserção periférica na economia brasileira. Além disso, pretende discutir as perspectivas futuras do Espírito Santo, a partir do papel decisivo que políticas públicas nas áreas de ciência, tecnologia e inovação assumem nesse início de século e ao mesmo tempo em que ficam claros os limites ambientais e de ocupação do espaço que o contemporâneo perfil produtivo do estado (voltado essencialmente para a produção de commodities) significa.

Palavras-chave: desenvolvimento regional; indicadores socioeconômicos; indicadores de ciência, tecnologia e inovação; Espírito Santo. 


\section{Abstract}

The objective of this paper is to show the contradictory character of the recent developments in the Espírito Santo's economy: an economy with indicators that reveal at the same time some degree of socioeconomic development and peripheral insertion in the Brazilian economy. In addition, plan to discuss future prospects of Espírito Santo, from the decisive role that public policies in the areas of science, technology and innovation are in this beginning of century and at the same time that are clear environmental limits and of occupation of space that the current production profile of the State (geared primarily for the production of commodities) means.

Keywords: regional development; socioeconomic indicators; science, technology and innovation indicators; Espírito Santo.

\section{Resumen}

El objetivo de este artículo es mostrar el carácter contradictorio de los recientes acontecimientos en la economía del Espírito Santo: una economía con indicadores que revelan al mismo tiempo cierto grado de desarrollo socio-económico y inserción periférica en la economía brasileña. Además, planea discutir las perspectivas de futuro del Espírito Santo, desde el papel decisivo que las políticas públicas en las áreas de ciencia, tecnología e innovación son en este principio de siglo y a la vez que son límites claro ambientales y de ocupación del espacio que significa el perfil actual de la producción del Estado (orientado principalmente para la producción de commodities).

Palabras clave: desarrollo regional; indicadores socio-económicos; indicadores de ciencia, tecnología e innovación; Espírito Santo. 


\section{Introdução}

A diversificação produtiva aparece como um dos principais objetivos das recentes tentativas de se planejar estrategicamente a economia do Espírito Santo por parte do Governo do Estado, como no caso do Plano de Desenvolvimento ES 2025. Mas a pergunta que surge neste contexto é: que tipo de diversificação produtiva a sociedade capixaba deseja?

Como se sabe, na dinâmica capitalista a diversificação a partir de atividades de Ciência, Tecnologia e Inovação (CT\&I) é fundamental. Porém, segundo Diniz (1993), algumas regiões do Brasil (basicamente o "polígono", que inclui áreas dos estados de Minas Gerais, São Paulo, Paraná, Santa Catarina e Rio Grande do Sul) apresentam uma melhor adaptação à Economia do Conhecimento. E, como mostraremos, a economia capixaba ainda não faz parte deste segmento verdadeiramente mais dinâmico da economia brasileira, apesar de várias medidas importantes do governo estadual nos últimos anos nas áreas de CT\&I.

Assim, por todos estes critérios, o Espírito Santo ainda é uma economia periférica em termos de geração de conhecimento e, neste caso, da sua incorporação ao processo produtivo. Situação que não deve se alterar no curto e médio prazos, pois os investimentos previstos para os próximos anos na economia capixaba são predominantemente de baixo conteúdo tecnológico, como mineração e siderurgia.

Dadas todas estas questões, o objetivo deste artigo é discutir que a economia capixaba apresenta um caráter contraditório pelo fato da mesma fazer parte do conjunto dos estados mais desenvolvidos por critérios socioeconômicos (Produto Interno Bruto - PIB - per capita, Índice de Desenvolvimento Humano - IDH, redução da pobreza, por exemplo), e do conjunto dos estados periféricos por outros (CT\&I); contradição esta que poderá se manifestar no futuro próximo ao se tentar manter os atuais níveis de crescimento e desenvolvimento da economia capixaba (que há várias décadas se mostram acima da média nacional). Pretende-se discutir também as perspectivas futuras do Espírito Santo, a partir do papel decisivo que políticas públicas nas áreas de CT\&I assumem na atual Era do Conhecimento.

Além desta introdução, o artigo é dividido nas seguintes seções: na segunda seção, é apresentada a literatura relacionada com o tema do artigo. A terceira seção traz um breve histórico recente da economia capixaba. A quarta, por sua vez, apresenta alguns indicadores socioeconômicos do Espírito Santo. A quinta seção apresenta vários indicadores sobre CT\&I para a economia local. Por fim, são apresentadas as conclusões do artigo.

\section{Elementos analíticos sobre a relação entre produção científica e tecnológica, desenvolvimento socioeconômico e eco- nomia regional}

Nesta seção apresentamos a literatura relacionada aos dois pontos sobre o Espírito Santo que se discutirá neste trabalho, a saber: i) análise da boa performance dos 
indicadores estaduais de bem-estar social, fruto do expressivo crescimento econômico local nos últimos anos; ii) avaliação do mal desempenho dos indicadores estaduais de ciência, tecnologia e inovação, desconexos dos resultados sociais e econômicos do Espírito Santo e que representam uma ameaça para a manutenção desses indicadores em patamares elevados no longo prazo.

Assim, quanto ao primeiro ponto citado anteriormente, segue revisão da literatura relacionada disponível. O Human Development Report - HDR (2001) sugeriu uma associação entre capacidade tecnológica e desenvolvimento humano dos países. Tal associação é sustentada por um conjunto de interações entre o desenvolvimento tecnológico e o desenvolvimento humano, por intermédio de múltiplos canais de influência, originando-se um circuito virtuoso entre o avanço tecnológico e o desenvolvimento humano.

Segundo o HDR (2001), inovações tecnológicas afetam o desenvolvimento humano de duas formas. Em primeiro lugar, disponibilizando produtos que afetam diretamente a saúde, a nutrição e as condições de vida das populações. Em segundo lugar, através de inovações tecnológicas que afetam indiretamente o desenvolvimento humano, em função de seu impacto sobre o crescimento econômico através de ganhos de produtividade.

Por outro lado, a influência do desenvolvimento humano no desenvolvimento tecnológico ocorre por meio do alcance de níveis mais elevados de educação, os quais constituem importantes fatores para a criação e a difusão de inovações. O Relatório destaca, ainda, que, com o desenvolvimento humano, existe uma maior disponibilidade tanto de cientistas para assumir atividades de pesquisa como de trabalhadores em condições de aprender e de dominar novas tecnologias.

Nessa linha, Machado et al. (2005) avaliaram as relações entre as produções científica e tecnológica e o nível de desenvolvimento humano no Brasil. As variáveis utilizadas foram: artigos científicos, patentes, IDH, infraestrutura urbana, infraestrutura cultural, educação, saúde e políticas de incentivo. Para a análise, foi utilizado o modelo Negative Binomial Hurdle. Os autores concluíram que:

i) há uma forte relação entre a infraestrutura cultural e as produções científica e tecnológica;

ii) há uma complexa associação entre as produções científica e tecnológica e os dados de bem-estar social. Os resultados dos testes realizados indicaram que as produções científica e tecnológica estavam associadas tanto a indicadores de desenvolvimento humano como a indicadores de presença e incidência de pobreza.

Por sua vez, quanto ao segundo ponto mencionado no começo desta seção sobre a avaliação dos indicadores estaduais de ciência, tecnologia e inovação, segue a literatura que aborda o tema. Cavalcante (2008) apresentou uma proposta de sistematização de teorias em economia regional cujo objeto de análise são espaços subnacionais sobre os quais há possibilidades concretas de intervenção e de levantamento de informações individualizadas.

Dessa forma, ele propôs uma sistematização do referido tema em três grandes blocos: o conjunto de te- 
orias clássicas da localização, as teorias de desenvolvimento regional com ênfase nos fatores de aglomeração e a produção recente em economia regional. Nesse último bloco, foram destacadas as abordagens que consideram os padrões de produção baseados na automação integrada flexível e da Nova Geografia Econômica.

Para o presente artigo, estamos interessados na produção recente em desenvolvimento regional, particularmente na abordagem que procura incorporar os fenômenos de reestruturação produtiva e aceleração da divisão internacional do trabalho. Com relação a este enfoque, Cavalcante (2008) apontou suas seguintes características gerais:

- $\quad$ uma grande ênfase dada à questão das externalidades (inclusive tecnológicas), com referências explícitas ao trabalho de Marshall (1890);

- uma forte influência do pensamento de Schumpeter $(1911 ; 1934 ; 1943)$ e da corrente evolucionista neo-schumpeteriana, sobretudo nos esforços de compreensão dos impactos dos processos de inovação tecnológica e aprendizado no desenvolvimento regional;

- $\quad$ uma ênfase nas relações não comerciais estabelecidas no âmbito das aglomerações, levando em conta aspectos de organização industrial e custos de transação.

Neste contexto, no debate sobre a inovação e seu papel no desenvolvimento econômico, as regiões ou localidades se tornam pontos de criação de conhecimento e aprendizado. Florida (1995), por exemplo, diz que regiões devem adotar os princípios de criação de conhecimento e aprendizado contínuo, devendo se tornar "regiões que aprendem".
Deixando um pouco de lado a parte teórica e partindo para caso empírico do Brasil, Cavalcante (2011) analisou a evolução das desigualdades regionais em CT\&I no país ao longo da última década. A revisão bibliográfica feita pelo autor reafirmou a associação entre as atividades de CT\&I e o desenvolvimento econômico e social e constatou-se a existência de elevados níveis de desigualdades regionais em CT\&I no Brasil.

Ademais, Cavalcante (2011) fez uma análise de estatísticas descritivas de indicadores regionalizados de CT\&I e calculou índices de desigualdades inter-regionais e interestaduais em CT\&I. Os principais resultados obtidos por ele foram:

a. O processo de convergência da base científica ao longo da década de 2000 foi lento;

b. A distribuição regional dos recursos do Conselho Nacional de Desenvolvimento Científico e Tecnológico (CNPq) e da Coordenação de Aperfeiçoamento de Pessoal de Nível Superior (Capes), proporcional à base instalada nos estados, não contribuiu para o processo de convergência da base científica;

c. De forma paradoxal, ao mesmo tempo em que se reduziram as desigualdades científicas, acentuaram-se as desigualdades regionais em esforços tecnológicos pelas empresas industriais.

Neste contexto merecem destaque também vários estudos (Diniz, 1993, por exemplo) que se referem à concentração que existe no Brasil de setores industriais mais intensivos em tecnologia numa área poligonal que vai da região central de Minas Gerais até o nordeste do Rio Grande do Sul, delimitada por Belo Horizonte-Uber- 
lândia-Londrina-Maringá-Porto Alegre-Florianópolis-São José dos Campos-Belo Horizonte.

Para que isso se concretize as regiões devem fornecer infraestruturas específicas que facilitem o fluxo de conhecimento, idéias e aprendizado, e que, ao mesmo tempo, tenham capacidade de governança local. Dado que o processo de inovação possui fortes componentes tácitos, cumulativos e localizados, os atributos regionais se tornam decisivos, daí surgindo a discussão do papel da inovação no desenvolvimento regional (Albagli, 1999).

Segundo estes autores, a região do polígono possui duas características marcantes:

a) concentra a maior parte das atividades de CT\&I e das firmas inovadoras do país;

b) em função disso, é a região que tem a inserção externa mais dinâmica no contexto regional brasileiro e, consequentemente, representa parcela substancial das exportações nacionais.

Ser dinâmico dentro da região poligonal significa apresentar taxas de crescimento industrial acima da média nacional, estimuladas pela interação entre ciência, tecnologia e inovação, ou seja, essencialmente por capacidades endógenas, e não por condições exógenas como incentivos fiscais, por exemplo. Essas são características dos estados mais desenvolvidos do ponto de vista produtivo, que apresentam indicadores de CT\&I relativamente altos em comparação com outros estados, como será mostrado a seguir.

Portanto, há relação clara entre desenvolvimento científico e tecnológico e melhores condições de vida para a população de uma região ou país. O que é interessante entender é por que, no caso do Espírito Santo, há tanta disparidade entre indicadores sociais e econômicos, de um lado, e científicos e tecnológicos, de outro, e como essa contradição pode afetar o futuro da economia capixaba e de seu potencial de crescimento e desenvolvimento econômico.

\section{Breve histórico da economia capixaba}

A economia do Espírito Santo foi relativamente bem estudada até o início dos anos 1990. Dentre os diversos trabalhos, cabe destacar: Rocha e Cossetti (1983); Almada (1984); Bittencourt (1987); Ferreira (1987); Rocha e Morandi (1991); Pereira (1998); e Rocha (1998). Estes trabalhos são de extrema importância para se entender o caminho percorrido pelo estado em seu desenvolvimento econômico até então. Porém, salienta-se que são poucos os estudos que abordam o cenário econômico local recente, destacando-se Mota (2002) e Bittencourt (2006).

Tais estudos permitem delimitar claramente dois grandes ciclos de desenvolvimento da econômica capixaba:

10 Ciclo: centrado na cafeicultura, sobretudo de base familiar, que predominou entre meados do século XIX e a década de 1950;

20 Ciclo: caracterizado pela industrialização, que teve uma $1^{a}$ fase (1960-1975) baseada no crescimento e instalação de pequenas e médias empresas e uma $2^{a}$ fase (1975-2000) baseada no crescimento e instalação 
de grandes empresas - os Grandes Projetos ${ }^{1}$ - produtoras de commodities.

Segundo o ES 2025, o ressurgimento da indústria petrolífera no Espírito Santo nos anos 2000 ensejou o início de um $3^{\circ}$ Ciclo de Desenvolvimento.

Como sintetiza a tabela 1 , elaborada de acordo com a tipologia da indústria proposta por Ferraz et al (1995), a economia capixaba passou por uma profunda mudança. Este processo visualiza-se o predomínio das commodities na produção industrial capixaba. Nesse contexto, vale ressaltar, com base em Magalhães (2011), que a economia do Espírito Santo sente mais intensamente os impactos de choques nos preços de commodities do que o Brasil e outros estados.

Essa mudança resultou de um substancial crescimento econômico, conforme tabela abaixo que mostra que o Espírito Santo cresce acima da média brasileira já há várias décadas. O resultado disso é que em 2009 o Espírito Santo tem o $11^{\circ}$ maior PIB brasileiro (ver tabela 7, a seguir), correspondendo a 2,06\% do PIB nacional, enquanto em 1970 correspondia a 1,2\% do PIB brasileiro (Mota, 2007, p. 93). Nesse sentido, Magalhães e Ribeiro (2011) apresentaram duas características marcantes da economia capixaba: i) o nível agregado de atividade vem apresentando, ao longo dos últimos 20 anos, uma tendência de crescimento sustentado; ii) as

$1 \mathrm{Na}$ literatura econômica capixaba, os termos Grandes Projetos e Grande Capital referem-se aos projetos industriais produtores de commodities realizados no estado entre meados dos anos 1970 e início dos 1980. Estes projetos compreenderam a construção da Aracruz Celulose, da Samarco e da Companhia Siderúrgica de Tubarão (CST), atualmente denominada ArcelorMittal Tubarão, bem como a ampliação das atividades da Companhia Vale do Rio Doce (CVRD). flutuações cíclicas do Espírito Santo são nitidamente mais voláteis do que as flutuações ocorridas no Brasil, com o estado tendendo a crescer acima da média nacional durante períodos de prosperidade, ao mesmo tempo que tende a crescer abaixo da média durante períodos de recessão.

Outro indicador importante refere-se ao desempenho no comércio exterior. Do lado as importações, em função do mecanismo do Fundo de Desenvolvimento das Atividades Portuárias (FUNDAP) e das trading companies, o Espírito Santo foi o 70 maior do país, representando 4,7\% das importações brasileiras em 2011, conforme tabela 3. Já do lado das exportações, o estado, em decorrência da produção de commodities, frequenta a lista dos principais estados exportadores, representando $5,9 \%$ do total das exportações brasileiras. Além disso, consoante Magalhães e Toscano (2010): (a) o grau de abertura estimado para o estado equivale a cerca de $49 \%$ do PIB ; (b) a economia estadual é, em média, cerca de duas vezes mais aberta ao comércio exterior em comparação à do país; e, conforme Pereira e Maciel (2010), (c) a economia capixaba é a $1^{\text {a }}$ no ranking de coeficientes de abertura das Unidades da Federação (UFs).

Contudo, essa participação relativamente alta das exportações capixabas deve ser vista com cautela quando se analisa sua composição, quase totalmente de commodities. Em 2011, por exemplo, a pauta de exportações era constituída da seguinte forma: pelotas de minério $(56,0 \%)$, produtos siderúrgicos $(11,8 \%)$, petróleo $(9,9 \%)$, celulose $(8,3 \%)$, café $(5,0 \%)$ e mármore 
Tabela 1: Participação relativa no VTI do Espírito Santo (1960-2010) (\%)

\begin{tabular}{l|c|c|c|c|c}
\hline Grupos de atividades & $\mathbf{1 9 6 0}$ & $\mathbf{1 9 7 0}$ & $\mathbf{1 9 8 5}$ & $\mathbf{1 9 9 6}$ & $\mathbf{2 0 1 0}$ \\
\hline Produtores de commodities & 8,1 & 17,9 & 49,9 & 53,5 & 69,8 \\
\hline Produtos duráveis de consume & 0,4 & 2,3 & 6,9 & 1,5 & 0,3 \\
\hline Indústrias tradicionais & 71,1 & 55,2 & 27,4 & 25,8 & 11,3 \\
\hline Indústrias difusoras de progresso técnico & 1,7 & 1,6 & 7,0 & 3,2 & 4,1 \\
\hline Sub-total & $\mathbf{8 1 , 3}$ & $\mathbf{7 7}$ & $\mathbf{9 1 , 2}$ & $\mathbf{8 4 , 0}$ & $\mathbf{8 5 , 6}$ \\
\hline Demais setores & 18,7 & 19,8 & 8,8 & 16,0 & 14,4 \\
\hline Total & $\mathbf{1 0 0 , 0}$ & $\mathbf{1 0 0 , 0}$ & $\mathbf{1 0 0 , 0}$ & $\mathbf{1 0 0 , 0}$ & $\mathbf{1 0 0 , 0}$ \\
\hline
\end{tabular}

Fonte: elaboração própria a partir de Pereira (1998, p. 135), para 1960; Mota (2002, p. 89), para 1970-1985; e IBGE (2012), para os anos de 19962010.

Tabela 2: Taxa média de crescimento real do PIB, Espírito Santo e Brasil (1960-2009) (\%)

\begin{tabular}{l|c|c|c|c|c}
\hline Itens & $1960 / 1970$ & $1970 / 1980$ & $1980 / 1990$ & $1990 / 2000$ & $\mathbf{2 0 0 2 / 2 0 0 9}$ \\
\hline Espíito Santo & 8,1 & 11,5 & 2,9 & 3,9 & 3,8 \\
\hline Brasil & 7,7 & 10,3 & 2,0 & 2,4 & 3,4 \\
\hline
\end{tabular}

Fonte: IPEADATA (2012b). Elaboração própria. 
Tabela 3: Comércio exterior dos estados brasileiros (2011)

\begin{tabular}{l|c|c}
\hline Estados selecionados & Participação (\%) nas importações & Participação (\%) nas exportações \\
\hline São Paulo & 36,3 & 23,4 \\
\hline Minas Gerais & 5,8 & 16,2 \\
\hline Rio de Janeiro & 8,4 & 11,5 \\
\hline Rio Grande do Sul & 6,9 & 7,6 \\
\hline Paraná & 8,3 & 6,8 \\
\hline Santa Catarina & 6,6 & 3,5 \\
\hline Espínito Santo & 4,7 & 5,9 \\
\hline Pará & 0,6 & 7,2 \\
\hline Bahia & 3,4 & 4,3 \\
\hline Demais estados & 19,0 & 13,6 \\
\hline Brasil & $\mathbf{1 0 0 , 0}$ & $\mathbf{1 0 0 , 0}$ \\
\hline
\end{tabular}

Fonte: MDIC (2012). Elaboração própria. 
e granito $(4,6 \%)$, totalizando $91,0 \%$ do valor exportado pelo estado naquele ano (MDIC, 2012). Esse predomínio das commodities na economia capixaba é claro, e tende a aumentar nos próximos anos, com a expansão prevista na produção de petróleo, além dos investimentos nas outras commodities que estão previstos (IJSN, 2012).

Portanto, os exemplos acima deixam claro que o padrão de crescimento da economia capixaba das últimas décadas foi fundamental para o desempenho acima da média brasileira que até 2009 é verificado, mas, ao mesmo tempo, apresenta nítidos limites em termos ambientais e de ocupação do espaço, que já começaram a aparecer, e que são preocupantes para o futuro. Assim, um novo perfil produtivo a partir da necessária diversificação da economia local tem que ser buscado. Mas antes veremos mais especificamente o que o perfil no período em estudo significou em termos de contribuição para a melhoria dos indicadores de crescimento e desenvolvimento econômico capixabas nas últimas décadas.

\section{Indicadores socioeconômicos do Espírito Santo: um estado entre os mais desenvolvidos do país}

O presente tópico apresenta dados de três indicadores socioeconômicos utilizados amplamente na literatura econômica para caracterizar o grau de desenvolvimento de países e regiões. Os três indicadores são: Índice de Desenvolvimento Humano (IDH), o PIB per capita e o percentual de pessoas pobres em relação à população total. Deve ficar claro também que o grau de desenvol- vimento do Espírito Santo será medido em comparação com o desempenho nos mesmos indicadores de outras Unidades da Federação brasileira.

Dessa forma, a tabela 4 mostra que os estados mais desenvolvidos do Brasil, segundo o IDH, possuem indicadores superiores ou iguais a 0,800 , segundo classificação do Programa das Nações Unidas para o Desenvolvimento (PNUD, 2005, p. 57). Assim, nota-se que o Espírito Santo já atingiu o patamar de alto desenvolvimento humano por estes critérios, estando na $7^{a}$ colocação entre todas as unidades da federação brasileiras. Passando ao PIB per capita, nota-se que com o crescimento das últimas décadas o Espírito Santo atingiu uma posição importante, sendo em 2009 o $6^{\circ}$ colocado neste ranking.

Ademais, dados da tabela 6, com base na Pesquisa Nacional por Amostragem Domiciliar (PNAD), mostram que no Espírito Santo ocorreu importante redução da taxa de pobreza ${ }^{2}$, de $28,08 \%$ em 2001 para $12,64 \%$ em 2009 - queda de $55,0 \%$ no período -, ao passo que na média nacional essa redução foi de $35,1 \%$. A partir desse resultado o estado saiu da $16^{a}$ posição do ranking nacional de menor taxa de pobreza, em 1970, para a 9a, em 2009, atrás de Santa Catarina (6,36\%), Mato Grosso do Sul $(10,05 \%)$, São Paulo $(11,01 \%)$, Distrito Federal $(11,32 \%)$, Goiás $(11,95 \%)$, Minas Gerais $(12,05 \%)$, Paraná $(12,37 \%)$ e Mato Grosso $(12,38 \%)$, enquanto essa taxa foi de $21,42 \%$ para o Brasil.

2 Percentual de pessoas na população total com renda domiciliar per capita inferior à linha de pobreza. A linha de pobreza aqui considerada é o dobro da linha de extrema pobreza, uma estimativa do valor de uma cesta de alimentos com o mínimo de calorias necessárias para suprir adequadamente uma pessoa, com base em recomendações da Food and Agriculture Organization (FAO) e da Organização Mundial da Saúde (OMS). 
Tabela 4: Índice de Desenvolvimento Humano (1970-2005)

\begin{tabular}{l|c|c|c|c}
\hline Estados selecionados & $\mathbf{1 9 7 0}$ & $\mathbf{1 9 9 1}$ & $\mathbf{2 0 0 5}$ & Ranking 2005 \\
\hline Distrito Federal & 0,652 & 0,799 & 0,874 & $1^{\circ}$ \\
\hline Santa Catarina & 0,477 & 0,748 & 0,840 & $2^{\circ}$ \\
\hline São Paulo & 0,643 & 0,778 & 0,833 & $3^{\circ}$ \\
\hline Rio de Janeiro & 0,657 & 0,753 & 0,832 & $4^{\circ}$ \\
\hline Rio Grande do Sul & 0,541 & 0,753 & 0,832 & $5^{\circ}$ \\
\hline Paraná & 0,440 & 0,711 & 0,820 & $6^{\circ}$ \\
\hline Espírito Santo & $\mathbf{0 , 4 1 5}$ & $\mathbf{0 , 6 9 0}$ & $\mathbf{0 , 8 0 2}$ & $\mathbf{7}^{\circ}$ \\
\hline Mato Grosso do Sul & 0,437 & 0,716 & 0,802 & $8^{\circ}$ \\
\hline Goiás & 0,404 & 0,700 & 0,800 & $9^{\circ}$ \\
\hline Minas Gerais & 0,412 & 0,697 & 0,800 & $10^{\circ}$ \\
\hline Piaui & 0,267 & 0,566 & 0,703 & $25^{\circ}$ \\
\hline Maranhão & 0,285 & 0,543 & 0,683 & $26^{\circ}$ \\
\hline Alagoas & 0,286 & 0,548 & 0,677 & $27^{\circ}$ \\
\hline Brasil & $\mathbf{0 , 4 6 2}$ & $\mathbf{0 , 7 4 2}$ & $\mathbf{0 , 7 9 4}$ & - \\
\hline
\end{tabular}

Fonte: IPEADATA (2012a) e CEPAL/PNUD/OIT (2008), para os dados de 2005. Elaboração própria. Nota: 2005 é o ultimo IDH disponível. 
Tabela 5: PIB per capita dos estados (1970-2009), em $R \$$ de 2000 (deflator implícito do PIB)

\begin{tabular}{l|c|c|c|c}
\hline Estados selecionados & $\mathbf{1 9 7 0}$ & $\mathbf{1 9 9 1}$ & $\mathbf{2 0 0 9}$ & Ranking 2009 \\
\hline Distrito Federal & 6.713 & 13.478 & 24.357 & $1^{\circ}$ \\
\hline São Paulo & 6.331 & 10.399 & 12.653 & $2^{\circ}$ \\
\hline Rio de Janeiro & 5.288 & 8.932 & 10.674 & $3^{\circ}$ \\
\hline Santa Catarina & 2.634 & 7.167 & 10.245 & $4^{\circ}$ \\
\hline Rio Grande do Sul & 3.684 & 7.765 & 9.551 & $5^{\circ}$ \\
\hline Espírito Santo & $\mathbf{2 . 1 0 0}$ & $\mathbf{5 . 9 3 6}$ & $\mathbf{9 . 2 4 5}$ & $\mathbf{6}^{\circ}$ \\
\hline Alagoas & 1.222 & 2.637 & 3.249 & $25^{\circ}$ \\
\hline Maranhão & 785 & 1.538 & 3.023 & $26^{\circ}$ \\
\hline Piauí & 624 & 1.645 & 2.922 & $27^{\circ}$ \\
\hline Brasil & $\mathbf{3 . 0 6 4}$ & $\mathbf{6 . 3 4 7}$ & $\mathbf{8 . 1 7 0}$ & - \\
\hline
\end{tabular}

Fonte: IPEADATA (2012a). Elaboração própria. 
Tabela 6: Percentual de pessoas pobres (1970-2009)

\begin{tabular}{l|c|c|c|c|c|c}
\hline Estados selecionados & $\mathbf{1 9 7 0}$ & $\mathbf{1 9 8 1}$ & $\mathbf{1 9 9 2}$ & $\mathbf{2 0 0 1}$ & $\mathbf{2 0 0 9}$ & Ranking 2009 \\
\hline Santa Catarina & 74,13 & 27,09 & 26,19 & 14,51 & 6,36 & $1^{\circ}$ \\
\hline Mato Grosso do Sul & 74,32 & 30,42 & 32,39 & 22,56 & 10,05 & $2^{\circ}$ \\
\hline São Paulo & 41,73 & 17,30 & 22,42 & 18,06 & 11,01 & $3^{\circ}$ \\
\hline Distrito Federal & 45,62 & 23,16 & 27,50 & 22,86 & 11,32 & $4^{\circ}$ \\
\hline Goiás & 77,79 & 40,61 & 31,86 & 25,27 & 11,95 & $5^{\circ}$ \\
\hline Minas Gerais & 77,50 & 35,02 & 35,13 & 25,00 & 12,05 & $6^{\circ}$ \\
\hline Paraná & 74,77 & 37,93 & 40,20 & 26,52 & 12,37 & $7^{\circ}$ \\
\hline Mato Grosso & 79,75 & 32,19 & 36,49 & 23,38 & 12,38 & $8^{\circ}$ \\
\hline Espírito Santo & $\mathbf{7 9 , 7 3}$ & $\mathbf{3 2 , 0 4}$ & $\mathbf{3 7 , 1 1}$ & $\mathbf{2 8 , 0 8}$ & $\mathbf{1 2 , 6 4}$ & $\mathbf{9}^{\circ}$ \\
\hline Rio de Janeiro & 26,34 & 24,67 & 26,14 & 22,49 & 13,48 & $10^{\circ}$ \\
\hline Rio Grande do Sul & 62,98 & 28,84 & 26,49 & 24,09 & 13,71 & $11^{\circ}$ \\
\hline Maranhão & 90,06 & 75,12 & 67,73 & 62,00 & 41,65 & $25^{\circ}$ \\
\hline Pemambuco & 84,19 & 60,32 & 65,37 & 58,13 & 42,24 & $26^{\circ}$ \\
\hline Alagoas & 88,14 & 62,31 & 63,08 & 63,01 & 47,70 & $27^{\circ}$ \\
\hline Brasil & $\mathbf{6 7 , 9 0}$ & $\mathbf{3 9 , 0 0}$ & $\mathbf{4 0 , 0 0}$ & $\mathbf{3 3 , 0 0}$ & $\mathbf{2 1 , 4 2}$ & - \\
\hline
\end{tabular}

Fonte: IPEADATA (2012a). Elaboração própria. 
Como visto a partir dos dados acima expostos, o Espírito Santo cresceu acima da média brasileira nas últimas décadas, o que possibilitou melhorias sensíveis no nível de vida da população, colocando o estado como um dos mais desenvolvidos do país. A seção seguinte analisará o perfil deste crescimento em termos de indicadores relacionados com o conhecimento, buscando identificar o que se pode esperar do processo de busca da mudança da economia capixaba nesse começo de século em termos de diversificação produtiva com maiores níveis de agregação de valor.

\section{Indicadores de CT\&I do Espírito Santo: o lado periférico da economia capixaba \\ 5.1. A situação da CT\&I no Espírito Santo no início do século XXI}

Como visto, ser dinâmico dentro da região poligonal que concentra os setores industriais brasileiros mais intensivos em tecnologia significa apresentar taxas de crescimento industrial acima da média nacional, estimuladas pela interação entre ciência, tecnologia e inovação. Essas são características dos estados mais desenvolvidos do ponto de vista produtivo, que apresentam indicadores de CT\&I relativamente altos em comparação com outros estados, como veremos agora.

Isto tudo pode ser confirmado a partir de estudos como o de Albuquerque et alli. (2002), que, com base em estatísticas de artigos científicos, patentes e pesquisadores, permitiu uma caracterização mais precisa das diferenças regionais de CT\&I no país. Com base nestas ideias foi elaborada a Tabela 7, que mostra alguns dados recentes sobre as diferenças regionais em termos de CT\&I. Em linhas gerais, os dados da referi- da tabela ratificam o principal resultado encontrado por aqueles autores, qual seja, a distribuição espacial das atividades científicas e tecnológicas do Brasil está concentrada no Centro-Sul do país, sobretudo nos estados que compõem a região do polígono. $\mathrm{E}$, assim como em Albuquerque et alli. (2002), os dados continuam evidenciando que esta concentração é superior à da atividade econômica.

Para se ter uma ideia mais precisa dessa concentração, foram totalizados os dados dos estados do polígono (SP, MG, PR, SC e RS) na Tabela 7. Esta região concentrou $89,3 \%$ das patentes concedidas no país (2011), $60,9 \%$ dos artigos científicos (2007-2010), 56,7\% dos pesquisadores (2010) e 58,9\% do PIB (2009). Quanto às demais regiões, notadamente as Regiões Norte e Centro-Oeste, as estatísticas confirmam a tese de Diniz e Gonçalves (2001) de "vazio e estagnação" em relação ao seu potencial para gerar atividade produtiva intensiva em conhecimento.

No que tange ao Espírito Santo, a Tabela 7 indica que o estado apresentou um desempenho discreto nas áreas de CT\&I, embora esteja geograficamente localizado na Região Centro-Sul do país. Ele representou apenas $0,3 \%$ das patentes concedidas, $1,0 \%$ dos artigos científicos, $1,2 \%$ dos pesquisadores, gerando $2,1 \%$ do PIB nacional. Tais dados são um indicativo de que a inserção capixaba no contexto regional brasileiro é pouco dinâmica do ponto de vista científico e tecnológico, o que fica ainda mais claro se for considerado que os estados do polígono, com exceção de Minas Gerais, apresentam participação no indicador de patentes ainda maior do que a do PIB no contexto da economia brasileira. 
Tabela 7: Participação de estados selecionados no total nacional de patentes*, artigos científicos**, pesquisadores*** e PIB, em \%

\begin{tabular}{l|c|c|c|c}
\hline Estados selecionados & $\begin{array}{c}\text { Artigos científicos } \\
(\mathbf{2 0 0 7 - 2 0 1 0 )}\end{array}$ & $\begin{array}{c}\text { Pesquisadores } \\
(\mathbf{2 0 1 0})\end{array}$ & $\begin{array}{c}\text { Patentes } \\
\mathbf{( 2 0 1 1 )}\end{array}$ & $\begin{array}{c}\text { PIB } \\
\mathbf{( 2 0 0 9 )}\end{array}$ \\
\hline São Paulo & 26,72 & 26,15 & 53,52 & 33,50 \\
\hline Rio de Janeiro & 10,92 & 12,73 & 7,31 & 10,90 \\
\hline Minas Gerais & 10,87 & 10,49 & 8,28 & 8,90 \\
\hline Rio Grande do Sul & 10,65 & 8,71 & 14,90 & 6,70 \\
\hline Paraná & 7,96 & 7,31 & 5,38 & 5,90 \\
\hline Bahia & 3,37 & 4,15 & 0,28 & 4,20 \\
\hline Distrito Federal & 2,64 & 3,08 & 0,97 & 4,10 \\
\hline Santa Catarina & 4,69 & 3,99 & 7,17 & 4,00 \\
\hline Pemambuco & 3,64 & 3,63 & 0,83 & 2,42 \\
\hline Goiás & 1,87 & 1,97 & 0,28 & 2,60 \\
\hline Espírito Santo & $\mathbf{0 , 9 9}$ & $\mathbf{1 , 1 5}$ & $\mathbf{0 , 2 8}$ & $\mathbf{2 , 0 6}$ \\
\hline Ceará & 2,54 & 2,26 & 0,41 & 2,00 \\
\hline Pará & 1,41 & 1,68 & 0,00 & 1,80 \\
\hline Amazonas & 0,94 & 1,34 & 0,00 & 1,50 \\
\hline Estados do polígono & $\mathbf{6 0 , 8 8}$ & $\mathbf{5 6 , 6 5}$ & $\mathbf{8 9 , 2 4}$ & $\mathbf{5 8 , 9 0}$ \\
\hline Brasil & $\mathbf{1 0 0 , 0 0}$ & $\mathbf{1 0 0 , 0 0}$ & $\mathbf{1 0 0 , 0 0}$ & $\mathbf{1 0 0 , 0 0}$ \\
\hline
\end{tabular}

* Patentes concedidas pelo Instituto Nacional de Propriedade Industrial (INPI).

** Artigos publicados por pesquisadores em periódicos nacionais, internacionais e em anais de eventos.

*** Pesquisadores com o título de doutorado.

Fonte: MCT (2012) e IPEADATA (2012b). Elaboração própria. 
Duas informações são importantes no sentido de se relativizar esses dados sobre patentes no Espírito Santo: de um lado, os dados sobre patentes depositadas nos Estados Unidos provavelmente mostrariam que a participação capixaba no total nacional é menor ainda que nas patentes depositadas no INPI, como sugerem os dados de Albuquerque et alli. (2002); por outro lado, as atividades inovativas podem estar sub-dimensionadas no estado pelo fato do principal produtor local de inovações, o Instituto Capixaba de Pesquisa e Extensão Rural (INCAPER), não registrar grande parte das mesmas sob a forma de patente.

\subsection{A situação da inovação no Espírito Santo: os dados da PINTEC (2008)}

A situação da inovação no Espírito Santo na primeira década do século XXI pode também ser entendida a partir dos dados da Pesquisa de Inovação Tecnológica (PINTEC). ${ }^{3}$ Para tal se usou indicadores de resultado (taxa de inovação) e indicadores de esforço (tipos de atividades inovativas desenvolvidas), tal como proposto por Cavalcante e De Negri (2010), além de indicadores de cooperação (tipos de cooperação realizada).

Durante o período 2006-2008, a PINTEC teve uma amostra de mais de 100 mil empresas do setor industrial do Brasil, segundo a Tabela 8 (que compara os treze estados mais desenvolvidos do Brasil em termos de participação no PIB nacional). Deste total, cerca de 3 O presente trabalho levou em conta somente os dados da PINTEC (2008), deixando de fora os dados das pesquisas de 2000, 2003 e 2005, porque seu objetivo é analisar as estatísticas mais recentes sobre inovação das empresas industriais capixabas e dos demais estados abrangidos pela pesquisa, e não a sua evolução.
38,3 mil ou $38,1 \%$ implementaram algum tipo de inovação - este percentual significa a taxa de inovação da indústria.

A indústria do Espírito Santo, apesar de ser relativamente tardia, apresentou taxas de inovação pouco abaixo da média brasileira no período 2006-2008. Neste período, a taxa de inovação da indústria capixaba permaneceu relativamente alta, $35,6 \%$, na $10^{a}$ posição do país - essa estatística, porém, será relativizada a seguir com a apresentação de outros indicadores. Por fim, o percentual de empresas industriais inovadoras capixabas em relação ao total do país atingiu 2,5\%.

A Tabela 9 mostra que das empresas industriais brasileiras que implementaram inovações entre os anos de 2006 e de 2008, 61,9\% o fizeram por meio de aquisição de máquinas e equipamentos. A segunda atividade inovativa mais utilizada foi o treinamento $(44,0 \%)$, seguida de projetos industriais $(24,1 \%)$. As atividades internas de Pesquisa e Desenvolvimento (P\&D), no entanto, foram empregadas por apenas $7,9 \%$ das firmas industriais que inovaram no período.

Esses dados comprovam a tese de que em países em desenvolvimento as inovações se dão muito mais pela compra - importação em alguns casos - de máquinas e equipamentos tecnologicamente mais avançados. $A$ atividade de $P \& D$, tida como uma das mais importantes fontes de inovação para autores como Freeman e Soete (1997), é realizada, proporcionalmente, por poucas empresas industriais no Brasil. Carneiro (2008) mostra como o Brasil ainda está longe de alcançar níveis de dispêndios em P\&D, como proporção do PIB, sequer comparáveis aos países com sistemas intermediários. 
Tabela 8: Empresas industriais que implementaram inovação, segundo Unidades da Federação (2006-2008)

\begin{tabular}{l|c|c|c|c}
\hline Unidades da Federação & Total (1) & Empresas que inovaram (2) & Taxa de inovação (3) & Part. relativa (4) \\
\hline Amazonas & 737 & 449 & 60,9 & 1,2 \\
\hline Pará & 1.581 & 433 & 27,4 & 1,1 \\
\hline Bahia & 2.967 & 1.083 & 36,5 & 2,8 \\
\hline Ceará & 2.085 & 840 & 40,3 & 2,2 \\
\hline Pemambuco & 2.312 & 729 & 31,5 & 1,9 \\
\hline Espírito Santo & $\mathbf{2 . 6 7 3}$ & $\mathbf{9 5 3}$ & $\mathbf{3 5 , 6}$ & $\mathbf{2 , 5}$ \\
\hline Minas Gerais & 12.578 & 5.208 & 41,4 & 13,6 \\
\hline Rio de Janeiro & 5.205 & 1.713 & 32,9 & 4,5 \\
\hline São Paulo & 33.962 & 12.379 & 36,4 & 32,3 \\
\hline Paraná & 8.534 & 3.641 & 42,7 & 9,5 \\
\hline Rio Grande do Sul & 9.127 & 4.029 & 44,1 & 10,5 \\
\hline Santa Catarina & 8.472 & 3.209 & 37,9 & 8,4 \\
\hline Goiás & 3.301 & 1.261 & 38,2 & 3,3 \\
\hline Sub-total & 93.534 & 35.926 & 38,4 & 93,8 \\
\hline Demais estados & 6.962 & 2.373 & 34,1 & 6,2 \\
\hline Estados do poligono & 72.672 & 28.466 & 39,2 & 74,3 \\
\hline Brasil & $\mathbf{1 0 0 . 4 9 6}$ & $\mathbf{3 8 . 2 9 9}$ & $\mathbf{3 8 , 1}$ & $\mathbf{1 0 0 , 0}$
\end{tabular}

Fonte: IBGE (2010). Elaboração própria

(1) Total de empresas industriais pesquisadas.

(2) Total de empresas industriais que implementaram inovações de produto e/ou processo.

(3) Percentual relativo de empresas industriais que implementaram inovações: $(2) /(1)=(3)$.

(4) Percentual relativo ao total de empresas industriais brasileiras que inovaram. 
Tabela 9: Empresas que implementaram inovações, segundo atividades inovativas desenvolvidas e Unidades da Federação (2006-2008)

\begin{tabular}{|c|c|c|c|c|c|c|c|c|c|c|c|c|}
\hline \multirow[t]{2}{*}{$\begin{array}{l}\text { Unidades da } \\
\text { Federação }\end{array}$} & \multicolumn{2}{|c|}{$\begin{array}{l}\text { Ativ. } \\
\text { interna de } \\
\text { P\&D }\end{array}$} & \multicolumn{2}{|c|}{$\begin{array}{l}\text { Aquisição } \\
\text { de } \\
\text { software }\end{array}$} & \multicolumn{2}{|c|}{$\begin{array}{l}\text { Aquisição de } \\
\text { máq. e } \\
\text { equip. }\end{array}$} & \multicolumn{2}{|c|}{ Treinamento } & \multicolumn{2}{|c|}{$\begin{array}{l}\text { Introd. das } \\
\text { inovações } \\
\text { tecnológicas } \\
\text { no mercado }\end{array}$} & \multicolumn{2}{|c|}{$\begin{array}{c}\text { Proj. } \\
\text { industrial e } \\
\text { outras prep. } \\
\text { técnicas }\end{array}$} \\
\hline & 1 & 2 & 1 & 2 & 1 & 2 & 1 & 2 & 1 & 2 & 1 & 2 \\
\hline Amazonas & 6,7 & 1,0 & 16,6 & 1,2 & 60,2 & 1,1 & 58,8 & 1,6 & 16,7 & 1,1 & 15,3 & 0,7 \\
\hline Pará & 2,5 & 0,4 & 19,3 & 1,3 & 57,5 & 1,1 & 26,1 & 0,7 & 23,5 & 1,5 & 25,6 & 1,2 \\
\hline Bahia & 4,4 & 1,6 & 29,6 & 5,0 & 53,7 & 2,5 & 48,8 & 3,1 & 24,2 & 3,8 & 38,9 & 4,6 \\
\hline Ceará & 2,7 & 0,7 & 26,8 & 3,5 & 65,6 & 2,3 & 31,7 & 1,6 & 33,8 & 4,1 & 11,4 & 1,0 \\
\hline Pemambuco & 9,1 & 2,2 & 16,0 & 1,8 & 52,6 & 1,6 & 19,3 & 0,8 & 8,5 & 0,9 & 16,7 & 0,3 \\
\hline Espírito Santo & 0,7 & 0,2 & 8,6 & 1,3 & 68,6 & 2,8 & 56,0 & 3,2 & 4,8 & 0,7 & 19,2 & 2,0 \\
\hline Minas Gerais & 5,9 & 10,2 & 15,1 & 12,1 & 68,2 & 15,0 & 45,9 & 14,2 & 14,6 & 11,1 & 28,2 & 15,9 \\
\hline Rio de Janeiro & 10,6 & 6,0 & 10,6 & 2,8 & 65,2 & 4,7 & 49,5 & 5,0 & 17,4 & 4,3 & 28,0 & 5,2 \\
\hline São Paulo & 9,6 & 39,2 & 18,5 & 35,2 & 54,8 & 28,6 & 45,5 & 33,4 & 21,6 & 39,0 & 25,6 & 34,4 \\
\hline Paraná & 8,2 & 9,9 & 17,2 & 9,6 & 64,4 & 9,9 & 47,2 & 10,2 & 13,0 & 6,2 & 23,9 & 6,4 \\
\hline $\begin{array}{l}\text { Rio Grande do } \\
\text { Sul }\end{array}$ & 7,6 & 10,2 & 17,0 & 10,5 & 67,7 & 11,5 & 39,3 & 9,4 & 17,4 & 10,2 & 22,4 & 9,8 \\
\hline Santa Catarina & 8,5 & 9,1 & 18,4 & 9,1 & 60,8 & 8,2 & 38,3 & 7,3 & 14,6 & 6,8 & 20,3 & 7,1 \\
\hline Goiás & 13,2 & 5,5 & 22,5 & 4,4 & 65,0 & 3,5 & 41,5 & 3,5 & 18,0 & 3,3 & 22,2 & 3,0 \\
\hline $\begin{array}{l}\text { Estados do } \\
\text { polígono }\end{array}$ & 8,3 & 78,6 & 17,5 & 76,5 & 61,0 & 73,2 & 44,1 & 74,5 & 17,8 & 73,3 & 24,8 & 73,6 \\
\hline Brasil & 7,9 & 100,0 & 16,9 & 100,0 & 61,9 & 100,0 & 44,0 & 100,0 & 17,9 & 100,0 & 24,1 & 100,0 \\
\hline
\end{tabular}

Fonte: IBGE (2010). Elaboração própria.

*Nota: considerou-se apenas as empresas que concederam grau de importância alto às atividades inovativas desenvolvidas.

(1) Percentual relativo ao total de empresas que implementaram inovações.

(2) Percentual relativo ao total de empresas que implementaram inovações no Brasil. 
Nas estruturas produtivas dos estados essa tendência não é muito diferente. Em unidades federativas como Espírito Santo, Rio de Janeiro, Minas Gerais, Rio Grande do Sul, Ceará e Goiás, a aquisição de máquinas e equipamentos foi feita por mais de $65 \%$ de suas empresas industriais pesquisadas, percentual superior à média verificada para o país. No caso das atividades internas de P\&D, a situação é ainda pior: Pará, Ceará e Espírito Santo apresentaram percentuais bem abaixo da média nacional. Além disso, em todas as atividades inovativas existe uma concentração muito grande nos estados mais desenvolvidos (SP, MG, PR, RS e SC) - acima de 78,6\% das firmas inovadoras do país em todos os casos.

Outro fator apontado como importante para a realização de inovações é a relação de cooperação empregada para tal. A Tabela 10 mostra que a taxa de cooperação é muito baixa na indústria brasileira, pois somente $10,1 \%$ das firmas industriais que realizaram alguma inovação cooperaram (ou, mais especificamente, concederam grau de importância alto e médio às parcerias) com outras organizações para tal fim. Dessas que cooperaram, $53,6 \%$ o fez com seus fornecedores, 35,3\% com seus clientes ou consumidores e 19,1\% com consultoria. As organizações menos usadas para a cooperação foram universidade e os centros de capacitação profissional.

Do ponto de vista regional, os dados evidenciam mais uma predominância dos estados mais desenvolvidos - aqueles que pertencem à região do polígono -, concentrando $82,5 \%$ das firmas do país que cooperaram para inovar. Em relação ao Espírito Santo, ele apresentou uma taxa de cooperação abaixo da média nacional $(4,4 \%)$. Contudo, $79,6 \%$ delas cooperaram com consul- toria e $35,3 \%$ com centros de capacitação, percentuais bem acima da média do país. $O$ destaque negativo no estado foi a baixa cooperação com universidades e institutos de pesquisa $(4,8 \%)$.

Em suma, todos esses dados da PINTEC (2008) sobre atividades inovativas mostram que a indústria do Espírito Santo, embora a princípio apresente uma taxa de inovação relativamente substancial, tem na verdade uma capacidade inovativa interna às firmas limitada. Isso porque parte expressiva de suas inovações se dá por meio de compra de máquinas e equipamentos, completando o quadro um baixo nível de empresas que realizaram P\&D e cooperação, notadamente com as universidades.

\subsection{A situação da inovação no Espírito Santo no $1^{\circ}$ decênio do século XXI: breve conclusão}

No que se refere às instituições de apoio à pesquisa e à inovação, somente muito recentemente o Espírito Santo passou a ter um arcabouço mínimo para o apoio ao desenvolvimento destas atividades (Mota, 2007). A Fundação de Amparo à Ciência e Tecnologia (FAPES) e a Secretaria de Estado de Ciência, Tecnologia, Inovação, Educação Profissional e Trabalho (SECTTI) surgiram em 2004, começando a funcionar plenamente em 2005, e o Núcleo de Inovação Tecnológica do Espírito Santo (NITES) foi criado em 2007. Estas instituições se juntaram ao Fundo Estadual de Ciência e Tecnologia (FUNCITEC), criado anteriormente, nos anos 1990. 
Tabela 10: Relações de cooperação das empresas que inovaram, segundo Unidades da Federação (2006-2008)

\begin{tabular}{l|c|c|c|c|c|c|c}
\hline $\begin{array}{l}\text { Unidades da } \\
\text { Federação }\end{array}$ & Total & $\begin{array}{c}\text { Taxa de } \\
\text { coperação } \\
(\%)\end{array}$ & $\begin{array}{c}\text { Clientes ou } \\
\text { consumidores } \\
(\%)\end{array}$ & $\begin{array}{c}\text { Fornecedores } \\
\mathbf{( \% )}\end{array}$ & $\begin{array}{c}\text { Consultoria } \\
\mathbf{( \% )}\end{array}$ & $\begin{array}{c}\text { Universidades } \\
\text { e inst. de } \\
\text { pesquisa (\%) }\end{array}$ & $\begin{array}{c}\text { Centros de } \\
\text { capacitação } \\
\text { profissional (\%) }\end{array}$ \\
\hline Amazonas & $\mathbf{5 8}$ & 12,8 & 25,9 & 50,8 & 22,1 & 40,9 & 14,2 \\
\hline Pará & $\mathbf{4 8}$ & 11,1 & 19,6 & 80,3 & 12,3 & 9,8 & 0,0 \\
\hline Bahia & $\mathbf{5 3}$ & 4,9 & 14,9 & 23,8 & 74,1 & 74,3 & 5,4 \\
\hline Ceará & $\mathbf{4 7}$ & 5,6 & 58,9 & 62,9 & 43,5 & 65,3 & 53,9 \\
\hline Pemambuco & $\mathbf{3 7}$ & 5,0 & 19,3 & 53,5 & 52,2 & 11,0 & 2,9 \\
\hline Espírito Santo & $\mathbf{4 2}$ & 4,4 & $\mathbf{8 , 7}$ & $\mathbf{2 1 , 8}$ & $\mathbf{7 9 , 6}$ & $\mathbf{4 , 8}$ & $\mathbf{3 5 , 3}$ \\
\hline Minas Gerais & $\mathbf{5 9 1}$ & 11,3 & 42,5 & 46,0 & 21,0 & 10,1 & 5,8 \\
\hline Rio de Janeiro & $\mathbf{1 1 9}$ & 7,0 & 37,7 & 50,4 & 18,1 & 20,6 & 12,6 \\
\hline São Paulo & $\mathbf{1 . 4 5 0}$ & 11,7 & 38,5 & 57,1 & 18,7 & 17,3 & 22,6 \\
\hline Paraná & $\mathbf{4 0 9}$ & 11,2 & 36,5 & 36,0 & 17,8 & 7,1 & 13,1 \\
\hline Rio Grande do Sul & $\mathbf{5 0 4}$ & 12,5 & 27,3 & 69,1 & 8,7 & 18,4 & 6,0 \\
\hline Santa Catarina & $\mathbf{2 3 4}$ & 7,3 & 31,9 & 64,0 & 12,4 & 32,9 & 27,0 \\
\hline Goiás & $\mathbf{1 0 1}$ & 8,0 & 13,1 & 51,5 & 12,6 & 10,5 & 5,0 \\
\hline $\begin{array}{l}\text { Estados do } \\
\text { polígono }\end{array}$ & $\mathbf{3 . 1 8 7}$ & 11,2 & $\mathbf{4 1 , 8}$ & $\mathbf{5 4 , 0}$ & $\mathbf{1 6 , 6}$ & $\mathbf{1 9 , 7}$ & $\mathbf{1 8 , 6}$ \\
\hline Brasil & $\mathbf{3 . 8 6 5}$ & 10,1 & $\mathbf{3 5 , 3}$ & $\mathbf{5 3 , 6}$ & $\mathbf{1 9 , 1}$ & $\mathbf{1 8 , 5}$ & $\mathbf{1 7 , 4}$ \\
\hline
\end{tabular}

Fonte: IBGE (2010). Elaboração própria.

Nota: considerou-se apenas as empresas que concederam grau de importância alto às parcerias de cooperação para inovar. 
Assim, ainda é muito cedo para se verificar algum impacto significativo dessas mudanças de diretrizes políticas sobre os indicadores de CT\&I capixabas. Porém, se levarmos em conta que o funcionamento de fundações de amparo à pesquisa e de secretarias de ciência e tecnologia é comum em todas as unidades da federação brasileira, não se nota nenhum avanço significativo que destaque as áreas de CT\&I capixabas frente a outras regiões brasileiras.

Neste sentido, considera-se que o governo estadual não possui ainda instrumentos adequados de planejamento estratégico para dar conta desta complexa tarefa. Uma de suas mais abrangentes ferramentas de planejamento, o Plano de Desenvolvimento ES 2025 (SEP, 2006), contava com apenas um dos seus 93 projetos estruturantes (o de número 58 ) diretamente voltado para as áreas de CT\&I, e mesmo assim de forma muito superficial. A própria meta estabelecida neste plano era muito genérica, ao vincular investimentos em CT\&I apenas em relação ao PIB capixaba.

Portanto, do exposto até aqui nesta seção, a partir da interpretação dos dados apresentados, tanto de patentes e artigos científicos, como da PINTEC, e levando-se em conta que o arcabouço institucional voltado para atividades de CT\&I ainda é muito recente, pode-se considerar que o sistema capixaba de inovação se encontra ainda num estágio embrionário de desenvolvimento (gerando pouca ciência, e menos inovação ainda, se comparadas aos $2 \%$ que o Espírito Santo ocupa no PIB brasileiro), revelando uma inserção pouco dinâmica e periférica da economia capixaba no contexto nacional quanto a estas atividades.

O resultado disso tudo é que, no que se refere à inovação, se considerarmos esta atividade como ir muito além da simples compra de máquinas e equipamentos mais modernos, chegando até ao desenvolvimento de P\&D, o Espírito Santo é movido por poucas "ilhas de excelência". Na indústria, por exemplo, há pouca atividade inovativa, concentrada em grandes empresas como a Fibria (antiga Aracruz Celulose) e menos ainda nos serviços e nos Arranjos Produtivos Locais - APLs (os principais APLs capixabas são os de mármore e granito, confecções, móveis e metalmecânico). Isso evidentemente é preocupante em termos de potencial de crescimento econômico com agregação de valor nos próximos anos.

\section{Considerações finais}

Como visto a partir de trabalhos como o Human Development Report - HDR (2001), existe associação clara entre capacidade tecnológica e desenvolvimento humano dos países e regiões. Tal associação é sustentada por um conjunto de interações entre o desenvolvimento tecnológico e o desenvolvimento humano, por intermédio de múltiplos canais de influência, originando-se um circuito virtuoso entre o avanço tecnológico e o desenvolvimento humano.

Dentro deste contexto, o presente artigo buscou analisar este importante tema com uma aplicação para a economia capixaba. Assim, o objetivo deste artigo foi analisar, em primeiro lugar, os indicadores de desenvol- 
vimento econômico e social do Espírito Santo, que em 2009 já apresenta níveis respeitáveis de PIB per capita, entre outros indicadores, que o colocam como um dos estados mais desenvolvidos do país. Para se chegar a esta situação, é inegável que a colocação em funcionamento dos Grandes Projetos, voltados para a produção de commodities, foi fundamental no processo de forte crescimento da economia estadual das últimas décadas.

Porém, o trabalho procurou mostrar que há um descompasso entre esta situação e a dos indicadores relacionados com conhecimento, nos quais o Espírito Santo continua sendo um estado periférico, fora do polígono que concentra a região mais dinâmica do país (evidentemente sem considerar possíveis benefícios futuros das medidas de política recentes do governo estadual, a partir das criações da FAPES e da SECTTI), o que pode representar perda de dinamismo no futuro quando a produção das referidas commodities enfrentar limites à sua expansão.

Analisando em perspectiva, o fato é que a CT\&I, na situação em que está, faz o Espírito Santo perder oportunidades, tanto na agregação de valor à produção local (dos seus APLs, por exemplo), como na atração de novos investimentos mais intensivos em conhecimento. Como visto, a economia capixaba atrai investimentos como a mineradora Ferrous, mas há alguns anos atrás se instalou em Vitória uma filial da Xerox, que por aqui ficou pouco tempo.

Ao mesmo tempo que perde oportunidades, a não consolidação de uma Economia do Conhecimento no Espírito Santo funciona como ameaça, pois estas atividades tendem a se concentrar nos estados mais desenvolvidos, dificultando no futuro a busca de agregação de valor com mais qualidade.

Considera-se que o fato de ser um estado pequeno não é empecilho para a colocação em prática de uma política mais agressiva para CT\&I, pois Santa Catarina é também um estado relativamente pequeno, mas que integra o polígono acima mencionado e que apresenta indicadores de inovação (patentes) razoavelmente acima da sua participação no PIB brasileiro, podendo ser um caso interessante em termos comparativos para a formulação de estratégias de políticas públicas capixabas na área de CT\&I.

No nível internacional, países também pequenos, como Irlanda e Israel, além das economias da Europa Nórdica, já vêm atingindo resultados econômicos expressivos a partir de investimentos orientados para o paradigma vigente das Tecnologias de Informação e Conhecimento. $\mathrm{O}$ caso da Finlândia é um exemplo interessante para o Espírito Santo, mesmo se considerarmos que este estado, por ser um ente subnacional, não pratica políticas cambial, monetária e aduaneira, como aquele país. Trata-se de um país de pequenas dimensões geográficas, condições climáticas adversas e com escassos recursos naturais, mas que tem na atividade de CT\&I um forte componente de seu crescimento econômico. A título de ilustração, o telefone celular, um dos produtos da contemporânea pauta de exportação finlandesa, tem entre 5 e 6 mil patentes.

Seguir de alguma forma os exemplos acima exigiria a produção industrial de base local incorporando 
minimamente o desenvolvimento de P\&D com equipes próprias, que para isso necessitariam de forte apoio institucional, tanto de agências públicas de fomento, como de universidades, que fornecem recursos humanos de qualidade e ao mesmo tempo são parceiras no desenvolvimento de novas tecnologias.

O resultado deste tipo de produção, como se sabe, são produtos de maior valor agregado, com salário médio mais alto, e inserção competitiva sustentável nos mercados nacionais e internacionais - vide De Negri et al (2005) para o caso brasileiro. Daí surgem empresas que se preocupam menos com situações macroeconômicas tradicionalmente adversas no Brasil (câmbio valorizado, juros e carga tributária altos, etc.), pois já conseguiram um grau de diferenciação que Ihes permite uma fixação de preços mais compensadora. Com isso, dependem também menos dos humores do mercado internacional, determinantes em muitos casos dos preços das commodities.

Este artigo considera, pelos dados apresentados na seção 5, que o Espírito Santo ainda tem espaço significativo para avançar em busca do salto crítico que permitiria o surgimento de empresas do tipo acima descrito em quantidade e qualidade na economia local. Empresas do perfil acima mencionado exigiriam a presença de recursos humanos de alto nível, a partir, por exemplo, de uma relação universidade-empresa profícua. E isso, infelizmente, ainda representa um grande gargalo da economia capixaba.

Considera-se que políticas públicas ainda mais ativas que as atuais, com medidas como o estabele- cimento de um detalhado planejamento estratégico para todas as áreas relacionadas com a CT\&I capixaba, maiores níveis de coordenação dos agentes, com mais recursos disponíveis (o crescente volume de royalties arrecadado pelo estado pode ser uma fonte interessante de recursos para estas áreas) e ao mesmo tempo avaliação dos resultados dos recursos aplicados, podem ser um interessante caminho inicial para que a CT\&I do Espírito Santo represente uma fonte relevante e sustentável de maiores níveis de agregação de valor para a economia local e de diversificação produtiva virtuosa. Isso tudo sem dúvida é essencial também para a manutenção ou até melhoria dos atuais indicadores socioeconômicos capixabas no longo prazo. 


\section{Referências Bibliográficas}

ALBAGLI, S. "Globalização e espacialidade: o novo papel do local". In: CASSIOLATO, J.E., LASTRES, H.M.M. (eds.). Globalização e inovação localizada: experiências de sistemas locais no MERCOSUL. Brasília: IBICT/MCT, 1999. cap. 5. ALMADA, V. F. P. Escravismo e transição: o Espírito Santo (1850-1888). Rio de Janeiro: Graal, 1984.

ALBUQUERQUE, E. M. et alli. "A distribuição espacial da produção científica e tecnológica brasileira: uma descrição de estatísticas de produção local de patentes e artigos científicos". Revista Brasileira de Inovação, Rio de Janeiro, v. 1, n. 2, jul.-set. 2002, p. 225-251.

BITTENCOURT, G.. A formação econômica do Espírito Santo: o roteiro da industrialização, do engenho às grandes indústrias. Rio de Janeiro: Cátedra, 1987.

História geral e econômica do Espírito Santo: do engenho colonial ao complexo fabril-portuário. Vitória: Multiplicidade, 2006.

CARNEIRO, A. P. M. "Estudo da importância da inovação tecnológica no Brasil através da PINTEC (Pesquisa Inovação Tecnológica/IBGE)". II Simpósio Internacional de Transparência nos Negócios, Niterói-RJ, 31 de julho a 2 de agosto de 2008. Disponível em: <http://www.latec.uff.br/transparencia/documentos/anais_transparencia2/T6_0111_0425. pdf>.

CAVALCANTE, L. R. Desigualdades regionais em ciência, tecnologia e inovação no Brasil: uma análise de sua evolução recente. IPEA, Brasília, 2011. Texto para discussão no 1.574.

. "Produção Teórica em Economia Regional: Uma Proposta de Sistematização". Revista Brasileira de Estudos Regionais e Urbanos, v. 02, p. 9-32, 2008.

CAVALCANTE, L. R.; DE NEGRI, F. "PINTEC 2008: análise preliminar e agenda de pesquisa". Radar Tecnologia, Produção e Comércio Exterior, Brasília, n. 11, p. 7-15, dez. 2010.

CEPAL/PNUD/OIT. Emprego, desenvolvimento humano e trabalho decente: a experiência brasileira recente. Brasília: CEPAL/PNUD/OIT, 2008.

DE NEGRI, J. A. et al. "Inovações, padrões tecnológicos e desempenho das firmas industriais brasileiras". In: DE NEGRI, João Alberto, SALERNO, Mário Sérgio (orgs.). Inovações, padrões tecnológicos e desempenho das firmas industriais brasileiras. Brasília: IPEA, 2005. cap. 1.

DINIZ, C. C. "Desenvolvimento Poligonal no Brasil: nem desconcentração, nem contínua polarização". Nova Economia, v. 3, n. 1, set. 1993.

, GONÇALVES, E. "Knowledge economy and regional development in Brazil". Les Troisièmes Journées de la Proximité - The Third Congress on Proximity, Paris, França, 13-14 dez. 2001. 
FERRAZ, J. C.; KUPFER, D.; HAGUENAUER, L. Made in Brazil: desafios competitivos para a indústria. Rio de Janeiro: Editora Campus, 1995.

FERREIRA, S. P.. Espírito Santo: dinâmica cafeeira e integração no mercado nacional - 1840-1960. 261 f. Dissertação (mestrado) - Universidade Federal do Rio de Janeiro, Instituto de Economia Industrial. 1987.

FLORIDA, R. "Toward the learning region". Futures, v. 27, n. 5, p. 527-536, 1995.

FREEMAN, C., SOETE, L. The economics of industrial innovation. 3. ed. London: Pinter, 1997.

INSTITUTO BRASILEIRO DE GEOGRAFIA E ESTATÍSTICA (IBGE). Pesquisa de Inovação Tecnológica (PInTEC) 2008. Rio de Janeiro: IBGE, 2010.

Pesquisa Industrial Anual (PIA) - 1996/2010. Disponível em: <www.ibge.gov.br>. Acesso em: 10 de outubro de 2012.

INSTITUTO JONES DOS SANTOS NEVES (IJSN). Investimentos previstos para o Espírito 2011-2016. Vitória-ES, 2012. Disponível em: <www.ijsn.es.gov.br>. Acesso em: 01 de outubro de 2012.

IPEADATA. Dados socioeconômicos dos estados e do Brasil para o período 1970-2011. Disponível em: <www. ipeadata.gov.br>. Acesso em: 01 de maio de 2012a.

. Dados nacionais e estaduais sobre PIB para o período 1960-2009. Disponível em: <www.ipeadata.gov. br>. Acesso em: 01 de maio de 2012b.

MACHADO, A. F.; ANDRADE, M. V.; ALBUQUERQUE, E. M. "Atraso tecnológico e atraso social: um modelo introdutório para investigar as relações entre as produções científica e tecnológica e o desenvolvimento humano no Brasil". Ensaios FEE, Porto Alegre, v. 26, n. 1, p. 471-504, jun. 2005.

MAGALHÃES, M. A. "Preços de commodities e nível de atividade em uma pequena economia aberta: evidências empíricas para o estado do Espírito Santo". Economia e Sociedade, v.20, n.3 (43), p. 533-566, Dez. 2011.

MAGALHÃES, M. A.; RIBEIRO, A. P. "Fatos estilizados dos ciclos de negócios no estado do Espírito Santo: uma abordagem quantitativa". Revista Econômica do Nordeste, v.42, n.3, p. 597-620, Jul.-Set. 2011.

MAGALHÃES, M. A.; TOSCANO, V. N. "Grau de abertura da economia do estado do Espírito Santo no período 1 otrim./04 - $2^{\circ}$ trim./09". Indicadores Econômicos FEE, v.37, n.4, p.225-240, 2010.

MARSHALL, Alfred. Princípios de Economia. São Paulo: Abril Cultural, 1982. Publicação original 1890.

MINISTÉRIO DA CIÊNCIA E TECNOLOGIA (MCT). Indicadores nacionais e estaduais de ciência e tecnologia: 2011. Disponível em: <www.mct.gov.br>. Acesso em: 01 de outubro de 2012.

MINISTÉRIO DO DESENVOLVIMENTO, INDÚSTRIA E COMÉRCIO EXTERIOR (MDIC). Estatísticas de comércio exterior por Unidade da Federação: 2011. Disponível em: <www.mdic.gov.br>. Acesso em: 01 de dezembro de 2012.

MOTA, F. C. M.. Integração e dinâmica regional: o caso capixaba (1960-2000). 161 f. Tese (doutorado) - Universidade Estadual de Campinas, Instituto de Economia, 2002. 
" "Avaliação da experiência capixaba de ciência, tecnologia e inovação". Parcerias Estratégicas, Brasília-DF, n. 25, dez. 2007, p. 93-114. PEREIRA, G. H. Política industrial e localização de investimentos e o caso do Espírito Santo. 1 ed. Vitória: EDUFES, 1998.

PEREIRA, L. V.; MACIEL, D. S. "O comércio exterior do estado do Espírito Santo". In: VESCOVI, A. P. V.; BONELLI, R. (Org.). Espírito Santo: instituições, desenvolvimento e inclusão social. Vitória, ES: IJSN, 2010. p. 95-137. PROGRAMA DAS NAÇÕES UNIDAS PARA O DESENVOLVIMENTO (PNUD). Relatório de Desenvolvimento Humano Brasil 2005. Disponível em: <www.pnud.org.br>. Acesso em: 01 de dezembro de 2009.

ROCHA, H. C.. Formação econômica do Espírito Santo e sua lógica empresarial. In: GUALBERTO, João, DAVEL, Eduardo. (orgs.). Inovações organizacionais e relações do trabalho: ensaios sobre o Espírito Santo. Vitória: EDUFES, 1998. p. 33-75.

, COSSETTI, Maria da Penha. Dinâmica cafeeira e constituição da indústria no Espírito Santo - 1850/1930. Vitória: UFES/NEP/COPLAN/IJSN/GERES, 1983.

MORANDI, A. M.. Cafeicultura e Grande Indústria: a transição no Espírito Santo 1955-1985. 1 ed. Vitória: Fundação Ceciliano Abel de Almeida, 1991.

SECRETARIA DE ESTADO DE ECONOMIA E PLANEJAMENTO (SEP). Espírito Santo 2025: plano de desenvolvimento. Vitória: SEP/MACROPLAN, 2006.

SCHUMPETER, J.. Business cycles: a theoretical, historical and statistical analysis of the capitalist process. New York: McGraw-Hill Books, 1964. Publicação original de 1911. 1934.

Teoria do desenvolvimento econômico. Rio de Janeiro: Fundo de Cultura, 1960. Publicação original de

Capitalismo, socialismo e democracia. Rio de Janeiro: Fundo de Cultura, 1961. Publicação original de 1943. UNITED NATIONS DEVELOPMENT PROGRAM. Human development report 2001: making new technologies work for human development. New York: UNDP, 2001. Disponível em: www.undp.org. 